\title{
AN INTRODUCTION TO SISTEM IDENTIFICATION FOR A QUARTER CAR PASSIVE SUSPENSION
}

\author{
Endang Susanti \\ Dosen Tetap Program Studi Teknik Elektro Universitas Riau Kepulauan
}

\begin{abstract}
At present, as a method of establishing mathematical model of the system, the system identification has been widely applied to the automatic control, aviation, spaceflight, astronomy, medicine, biology, marine ecology and society, economics and many other fields. With the rapid development of science and technology, the status of system identification technique in various disciplines is becoming increasingly important. This paper introduces an system identification used for a quarter car passive suspension and a review previous research.
\end{abstract}

\section{Introduction}

The great interests in the vehicle research field is improving the car ride quality and handling performance. The performance improvement can be done by design and analyze the car suspension system controller. Thus the high fidelity mathematical model capturing realistic dynamic ofthe car suspension system is necessary (Hanafi, 2009). Identification system is the best way to solve mathematical model in a system (Zadeh, 1961). System identification is to make some kind of mathematical models under a certain purpose from the measurements of the input and output data of the dynamic system (Mukai, 2010; Ling et al; 2011; Chen 2011). System identification includes linear system identification and nonlinear system identification (Lifu, 2013). While linear time invariant systems can be characterized by their impulse response, frequency responses, transfer function or state equations, the characterization of nonlinear dynamic systems is considerably more complex since all system that are not linear are contained in this class. Also, many well known methods exist for the system identification of linear time invariant systems characterized in any of the above forms (Ljung, 1987).

\section{Modeling}

Modeling and system identification methods are needed for the interpretation of observations and measurements obtained from some systems of study (Ljung, 1987). As models constitute the necessary link between experiments and decision making (for example, prediction, learning new rules, and data compression), modeling and identification are very important for all applied science and engineering 
Modeling is the first step of system identification from experimental data. Modeling is started from a set of model components and gives rise to a physical model with some network (system) structure. The resultant network (system) behavior can be determined from the network (system) structure and from the properties of the balance equations between all interacting components (Chen, 2010).

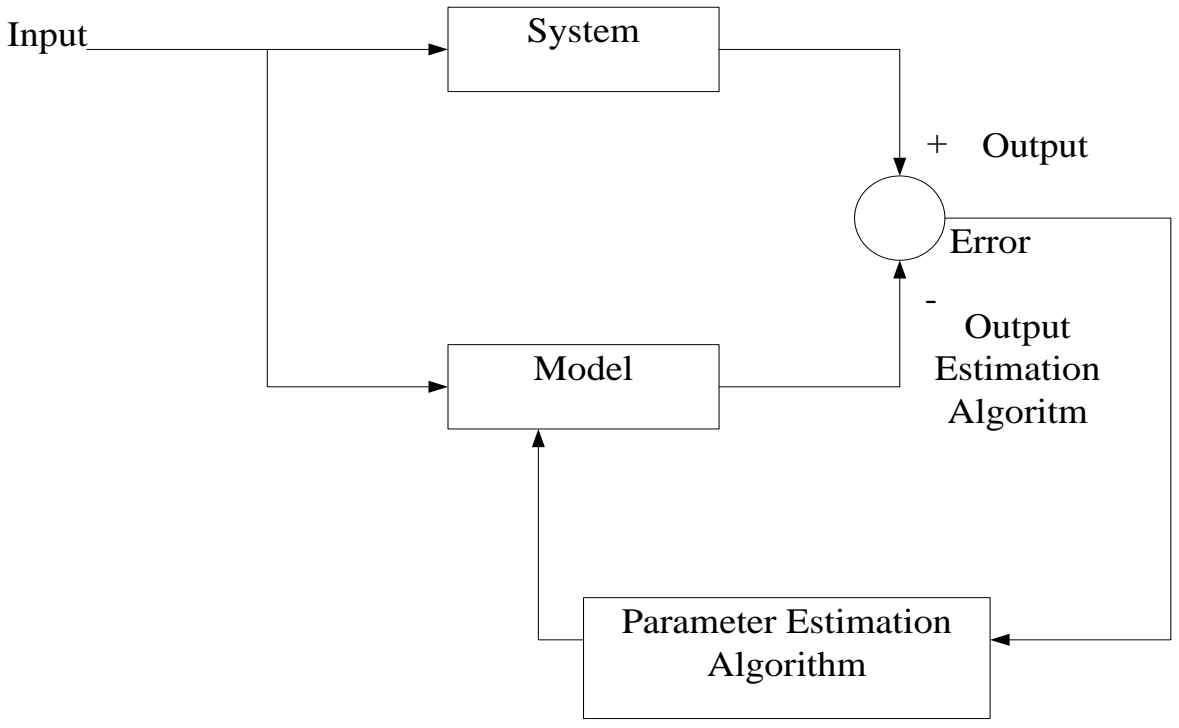

Figure 1.1 The structure of system identification

For more advanced applications, it may be necessary to use model that describe the relationships among the system variable in terms of mathematical expressions like difference or differential equation we shall call such models mathematical (or analytica) models (Ljung, 1987).

A mathematical model can be defined as a set of relationships between the phissical variables in the system to be modeled. These relation are mapped by mathematical structure of algebraic equation, differential equations or difference equation. In analysis a mathematical model of the system can be used to investigate how a unforeseeable event could affect the system (Maria, 1997)

\section{A Vehicle Suspension System}

Vehicle suspension is a mechanism located between the sprung mass (vehicle body) and unsprung mass (wheel) of the vehicle. The suspension provides force 6 between two masses of the vehicle according to certain state variables of the vehicle. The purposes of the suspension are to support the vehicle weight, keep the wheels on the ground, minimize the 
transient force to the body, maintain good ride comfort, and enhance handling performance (Tan and Bradshaw, 1997; Du and Li et al, 2012).

Suspension system is one of the critical components in the present of vehicle system. Ride safety and the handling capabilities of vehicle are mainly determined by its suspension system, which transmits the forces between the vehicle and the road. Suspension consists of the system of springs, shock absorbers and linkages that connects a vehicle to its wheels (Ghazaly and Moaaz, 2014; Qamar and Khan, 2013; Xu and Fei, 2010).

Active suspensions can continuously change the energy flow to or from the system when required. Furthermore, characteristics of active suspensions can adapt to instantaneous changes in driving conditions detected by sensors. As a result active suspensions can improve both riding comfort and handling performance to satisfactory levels (Kastiban and Pourqorban et al 2009, Hassanzadeh and Alizadeh, 2010; Xu dan Fi, 2010; Chenyu and Shuo, 2013).

The passive vehicle suspension system consists of springs and dampers usually placed parallel to one another at each corner of the chassis of the vehicle. Their main purpose is to either store or dissipate energy There are primarily two types of external disturbances, road and load disturbances (Jhon, 2011). Passive suspension systems include the conventional springs and shock absorbers used in most cars. The springs are assumed to have almost linear characteristics, while most of the shock absorbers exhibit nonlinear relationship between force and velocity. In passive systems, these elements have fixed characteristics and hence, have no mechanism for feedback control (Esmalizadeh, 1996; Gellips, 1992).

A high fidelity analytical model not only would help to solve such wheel control and harshness problem, but also could optimize the overall system design. The need for such a mathematical model capable of effectively capturing the highcfrequency system dynamics has been stressed in recent years (Tan and Bradshaw, 1997; Hanafi, 2006).

\section{The Car Passive Suspension}

The vehicle passive suspension system consists of springs and dampers usually placed parallel to one another at each corner of the chassis of the vehicle. Their main purpose is to either store or dissipate energy. There are primarily two types of external disturbances, road and load disturbances (Jhon, 2011). The passive suspension systems include the conventional springs and shock absorbers used in most cars.

The springs are assumed to have almost linear characteristics, while most of the shock absorbers exhibit nonlinear relationship between force and velocity. In passive systems, these 
elements have fixed characteristics and hence, have no mechanism for feedback control (Esmalizadeh, 1996). According to Gillespie, 1992 Passive suspension consist of convential components with spring and damping (shock absorber) propertis which are time-invariant. Passive elements can only store energy for some portion of a suspension cycle (spring) or disspate energy (shock absorber) no external energy is direcly supplied to this type of suspension.

\subsection{Spring Element}

The springs support the weight of the vehicle, maintain ride height, and absorb road shock. Springs are the flexible links that allow the frame and the body to ride relatively undisturbed while the tires and suspension follow the bumps in the road. Springs are the compressible link between the frame and the body. When an additional load is placed on the springs or the vehicle meets a bump in the road, the springs will absorb the load by compressing. The springs are a very important component of the suspension system that provides ride comfort. Shocks and struts help control how fast the springs and suspension are allowed to move, which is important in keeping tires in firm contact with the road.

Most of the spring element in independent suspension has a coil. A coil spring is a length of spring steel rod wound into a spiral form as shown in Figure 2.1. The deformation of spring is directly proportional to the mechanical force or torque applied to it.

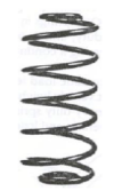

Figure 1: Coil spring elements

The movement of a spring is called oscillation, and the stiffness of a spring is referred to as spring rate. For linear rate springs they have equal spacing between the coils, and one basic shape with a consistent wire diameter. Figure 2 shows the schematic of coil spring.

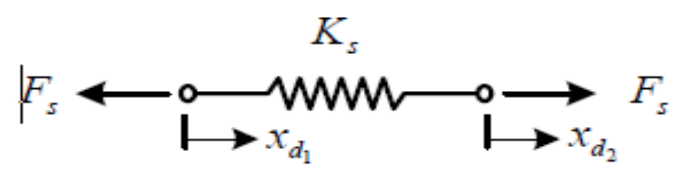

Figure 2: A physical model of a spring 
The dynamic formulation of a linear spring is shown in equation (2.1)

$$
F_{s}=K_{s}\left(X_{d_{2}}-X_{d_{1}}\right)
$$

Where $K_{s}$ is spring coefficient or spring rate, $F_{S}$ is applied fore and $X_{d_{1}}$, $X_{d_{2}}$ are displacements.

\subsection{Damping Element}

A damping element or a shock absorber is a mechanical element that dissipates energy in the form of heat. The force associated with a damper is proportional to the velocity difference of both ends. Figure 3 shows the mechanical damper element.

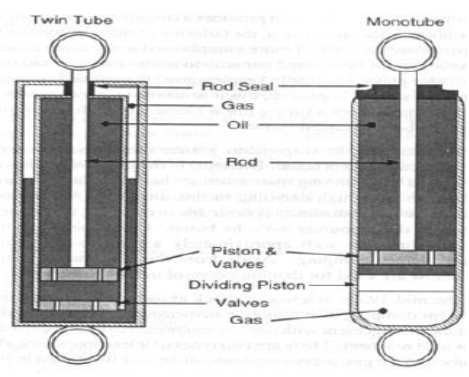

Figure 3: A damper element

The damper physical model related to the force as shown in figure 4

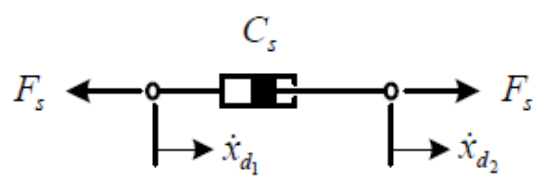

Figure 4: A physical model of damping

Equation (2.2 ) show the mathematical expression of linear force for damper dynamic,

$$
F_{s}=C_{s}\left(\dot{x}_{d_{2}-} \dot{x}_{d_{1}}\right)
$$

Where $C_{s}$ is a damping coefficient, $F_{s}$ is applied force and $\dot{x}_{d_{1}}, \dot{x}_{d_{2}}$ are volties.

\subsection{Tyre Dynamic}

High frequency dynamic tyre models for predicting the tyre response on uneven road surfaces are major elements of current state of the art ride comfort and handling simulations. They provide the interface between the vehicle and the road surface (Schmeitz and Pauwelussen, 2000). Mancosu et al. (1999) proposed the rigid ring model of tyre to offer a good balance between computational effort and accuracy. The valid frequency range of the rigid ring model is $0-80 \mathrm{~Hz}$ 
Tan and Bradshaw (1997) modeled the tyre as ten equality spaced vertical spring across the tyre contact patch with a point follower. This model was found to model the wheel hub acceleration very accurately over the impact bump. This model is used to duplicate the high frequency tyre force from an impact bump.

A tire is composed of high modulus flexible filaments such as textile, metal or glass, embedded in and bonded to a low modulus matrix (rubber or rubber like polymer) (Miege, 2002).

The tyre has three main components:

1. Tyre carcass: flexible filaments of high modulus cord, natural textile, synthetic polymer, and glass fiber or fine hard drawn steel. There are usually multi filaments layers or piles.

2. Tyre casing: the layer of the high modulus cord or filaments are turned around bead coils made of a number of high tensile, hard drawn steel wire, located at the inner edge of the tire sidewalls.

3. Tyre tread: the only part of the tyre in contact with the road. It protects the casing and provides frictional contact to transmit driving, braking and corning forces.

\section{System Identification}

System identification as identification in the determination on the basis on input and output of a system to which the system to which the system under test equivalent (Zadeh, 1967; Tan, 1997). System identification is to make some kind of mathematical models under a certain purpose from the measurements of the input and output data of the dynamic system (Mukai, 2010).

From data involves three basic entities (Ljung,1987) :

1. The data record. The input output data are sometimes recorded during a specifically designed experiment. The object with experiment design in thus to make these choices to contraints that may be at hand. In other cases the user may not have the possibility to effect the experiment, but must use data from normal operation system.

2. The set of the models. A set of candidate models is obtained by specifying within which collection of models where a suitable one is looking for. This is no doubt the most important and, at the same time, the most difficult choice of the system identification procedure. It is here that a priori knowledge and engineering intuition and insight have to be combined with formal properties of models. Sometime the model with some 
unknown physical parametersis contructed from basic physical laws and other well estabilished relationship.

3. Model validation is a step a determine the best modeinthe set, guided by the data. The assessment of model quality is typically based on how the models perform when they attempt to produce the measured data.

The system identification procedure, with the aim to arrive at an appropriate mathematical model of the system, is described in some detail as in figure 1.2. As mentioned before, prior knowledge, objectives, and data are the main components in the system identification procedure, where prior knowledge has a key role. It should be realized that these entities are not independent. Most often, data is collected on the basis of prior system knowledge and modeling objectives, leading to an appropriate experiment design. At the same time observed data may also lead to an adjustment of the prior knowledge or even to the objectives (Keesman, 2011).

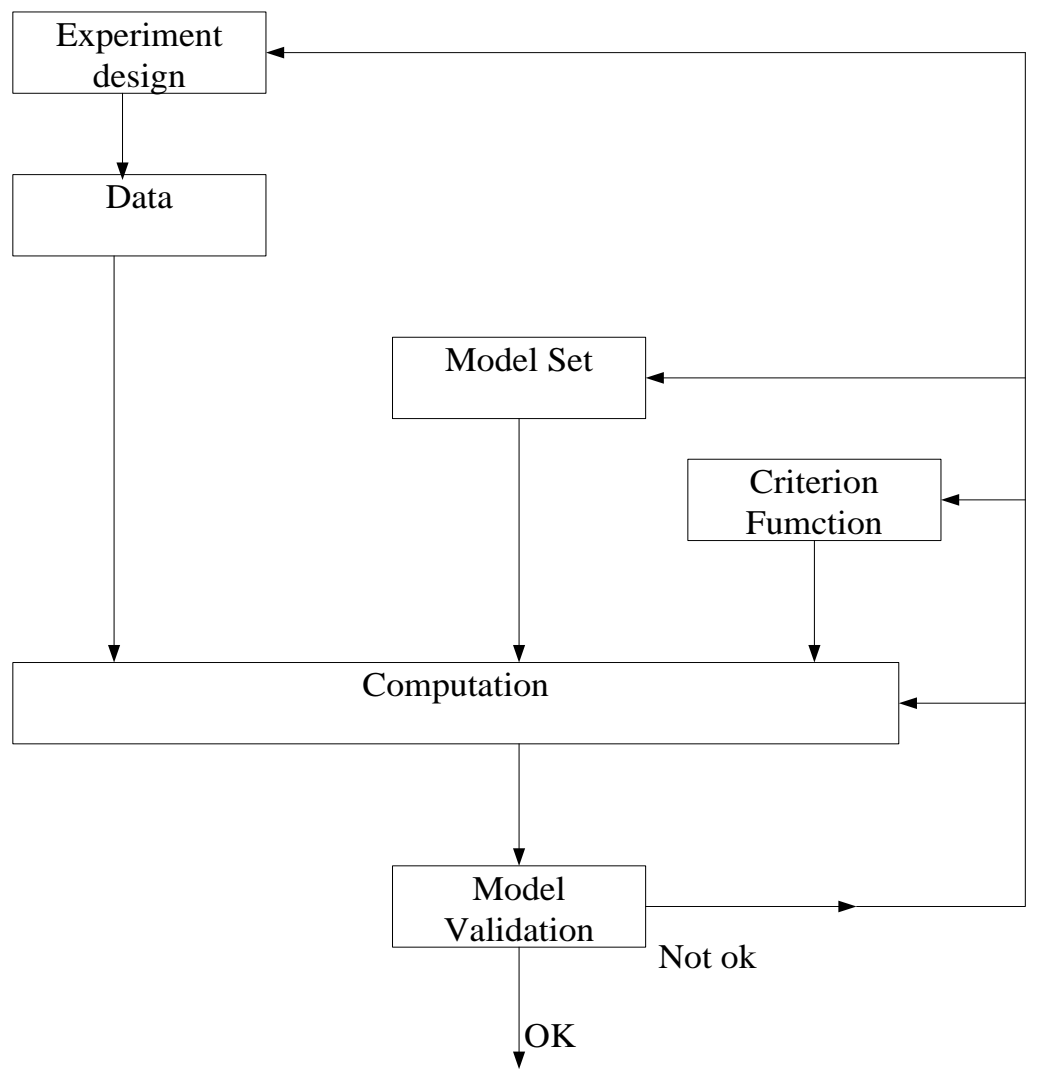


Figure 5. The system indentification loop

Figure 1.2 shows that the choice of a model set is completely determined by our prior knowledge of the system. This choice of a set of candidate models is without doubt the most important and most difficult step in a system identification procedure.

Recently, convex optimization has been successfully demonstrated as applied to nonlinear system identification (Fack, 2013; Yonghehan, 2012; Abdullah, 2012, Ljung, 2014). Convex optimization is the basis for several heuristics for solving nonconvex problems (Boyd, 2004). Convex optimization can be used to solve very large, practical engineering problems reliably and efficiently. It provides untraditional computational and mathematical tools, which can easily be used in order tosolve not only problems such as least squares and linear programming but also a much complicated and real-life problem (Hindi, 2004; Boyd, 2004; Amuetze, 2005; Browin and Lewis, 2009; Abdullah 2012).

\section{$5.1 \quad$ The Data}

In many studies, data were collected in laboratory environment and others from real experimental test of system. Some kinds of sensor presented to measure input output data.

Majjad (1997) collected the data in laboratory. The road profile considered as the input variable and described as a random function, and its elevation is approximated by a Gaussian white noise. There are two output data variables, i.e. vertical body acceleration and suspension deflection. The vertical body acceleration is measured using a vertical accelerometer sensor and fixed on the top of suspension. The suspension deflection is measured with a displacement sensor

Kim and Ro (2000) did laboratory test to generate step function signal as input variable. Output variable is suspension displacement and measured with displacement sensor.

Hanafi (2005), Hanafi (2009), Hanafi and Fua'ad, (2009), Hanafi (2010) measured input output data on real experimental test. The input variable is the unsprung mass position and measured by installing a vertical accelerometer sensor on the in front shaft at the down of suspension. The position is produced by twice integration of sensor output. The vertical body as output variable is sensed by put a vertical accelerometer sensor on the top of suspension.

\subsection{A Set of Candidate Model}


Applying physics law, mathematical model of system is derived from its physical model. Sometime, this model is used to approximate and predict the structure and parameter of candidate model. This mechanism has been extensively used in last work, among:

Majjad (1997) generated two dynamic equations from his a quarter vehicle physical model. These equations are continuous time and have nonlinear component. Based on them, a set of candidate model is constructed in ARX model.

Kim and Ro (2000) formulated a set of candidate model in ARX structure. Model assumed continuous time

Hanafi and Fua'ad (2005) formulated a set of candidate model in nonlinear hammestein structure. Hanafi and Fua'ad et al; (2009), Hanafi (2009), Hanafi (2010) derived the Nonlinear AutoRegressive with eXogenous input (NARX) structure as a set of models.

\subsection{Estimation Parameter}

In system identification technique, the parameter of model will be estimated applying some criteria of estimation method. There are have been widely used to identify a quarter vehicle model, among-other.

Majjad, 1997 estimated the vehicle suspension parameters employing nonlinear identification. Estimation method is based on Recursive Least Square (RLS) algorithm. The first step to determines the suspension velocity by applying numeric method. The estimating parameter is sprung mass of the vehicle. The idea here is to discern also two procedures to estimate the force. It will estimate by two models, model first needs only two measurements (the body acceleration and suspension displacement). Second model used three measurements: vertical acceleration of tire and body and suspension deflection. System identification derived in two ways; first way estimated the linear piece damper characteristic. Second way estimated of the nonlinear damper characteristic. These parameters are fundamental for a realistic suspension control design. The result is shown that only used to sensors and using Recursive Least Square the nonlinear damper characteristic can be good estimate.

Kim and Ro (2000) used Least Square estimation method to estimate a quarter car dynamic model. The identification result shows that 2 mass model is effective to representing a quarter vehicle model and give an accurate set of identified parameter

Hanafi and Fua'ad (2005) used Multilayer Perceptron Neural Network (MPNN) to estimate nonlinear model of a quarter vehicle dynamic. Model structure is assumed has a 
nonlinear component and constructed in Hammestain structure and Nonlinear AutoRegressive with eXogenous input (NARX)

\section{REFERENCES}

Aly, A., A., and Salem, F., A. (2013). Vehicle Suspension System Control: A Review. International journal Of Control, Automation And System, Vol. 2, No. 2, July 2013

Experimental Approach of a Quarter Car Modelling. UTHM, Johor, Malaysia

Chen, T ., Andersen, S., M, and Ljung, L. (2014). System Identification Via Sparse Multiple Kernel-Based Regularization Using Sequential Convex Optimization Techniques. IEEE Transaction On Automatic Control, Vol 59, No. 11, November 2014.

Esmalizadeh, E., and Taghirad, H.D. (1996). Active Vehicle Suspension With Optimal State Feedback Control. International Journal of Mechanical

Falk, T. (2013). Nonliear system Identification Using Structured Karnel Based Model. PhD Thesis. Katholieke Universiteit Leuven.

Fu, L., and Li, P. (2013), The Research Survey of System identification Method, Fifth International Conference on Intelligent Human-Machine Systems and Cybernetics.

Ghazali, M., N., and Moaaz, O., A. (2014), The Future development and Analysis of vehicle Active Suspension System, IOSR Journal of Mechanical and Civil Engineering (IOSR-JMCE), Volume 11, Issue 5 Ver (SepOct 2014, PP 19-25

Gillespie, T. D. (1992). Fundamentals of Vehicle Dynamics. Society of Automative Engginer. Inc, Warrendale.

Hanafi, D. (2009). The Quarter Car Fuzzy Controller Design Based on Model from Intelligent System Identification. IEEE Symposium Electronics and Aplication (ISIEA 2009). Oktober 4-6, Kuala lumpur, Malaysia

Hanafi, D. (2010). PID Controller Design For Semi Active Car Suspension Based On Model From Intelligent System Identification. Second International Conference on Computer Engineering and Applications.

Hanafi, D and Rahmat, F., M., (2005) System Identification Of Hammerstein Model A Quarter Car Passive Suspension System Using Multilayer Perception Neural Network (MPNN), Jurnal Teknologi, Universiti Teknologi Malaysia, 2005, 95-109

Hanafi, D., Rahmar, B. F., Ahmad, B. A. Z., and Zaid, M.B.A. (2009). Intelligent System identification for an Axis of Car Passive Suspension System Using Real Data. Journal IEEE, 2009

Hindi, H. (2004). A Tutorial on Convex Optimization. Proceeding of the American Conference, Boston, Massachusetts, June 30-July 2, 2004

John, S., Pedro, J., O., and Roza, C., R. (2011) Enhanced Slip Control Performance Using Nonlinier Pssive Suspension System. IEEE/ASME International Confenence on Advanced Intelligent Mechatronics (AIM2011), Budapet, Hungary. July 3-7, 2011

Kashtiban, M., A., and Pourqorban, N., et al. (2009), Nonlinier Optimal Control of a half Car Active Suspension. Second International Conference on Computer and Electrical Engineering, 2009

Keesman, K., J. (2011). System Identification an Introduction, Springer, London 2011

Kim, C., and Ro, P.I. (2000). Reduced Order Modeling and Parameter Estimation for A Quarter Car Suspension System. Instrumentation Mechanical Engineering 214(D)

Liberti, L. (2008). Introduction to Global Optimization, Februari, 15, 2008, France.

Ljung, L., (1987). System Identification : Theory for the user, Prentice-Hall, Inc, New Jersey

Luo, Z., and Yu,W. (2006). An Introduction to Convex Optimization Fpr Communication and Signal Processing. IEEE Journal In Communication, Vol 24, No.8, August 2006

Majjad, R. (1997). Estimation of Suspension Parameters. Proceedings of IEEE International Conference on Control Applications, 552-527, Hartford.

Mukai, F., Adachi. S., Baba, T., and Kamiyama, N,. (2010). An Application of System Identification Theory to The Ultrasound Doppler Image Processing System. SICE Annual Conference, August 28-21, 2010, Taipei, Taiwan.

Tan, H-S., and Bradshow, T. (1997). Model Identification of an Automotive Hydraulic Active Suspension System. Procceding of The Amarican Control Conference, New Mexico, June, 1997

Ujang, B., C., Jahanchahi, C and Took, C., C. et al. (2014) Adaptive Convex Combination Approach for the Identification of Improper Quaternion Processes. IEEE Transactions on Neural Networks and learning systems, Vol. 25, No.1, January 2014.

Zadeh, L., A. (1961). From Circuit Theory to System Theory. Proceedings Of The IRE, August 25, 1961. 
